\title{
PROCEEDINGS OF THE EIGHTEENTH ANNUAL MEETING OF THE AMERICAN SOCIETY FOR CLINICAL INVESTI- GATION HELD IN ATLANTIC CITY, NEW JERSEY, MAY 3,1926
}

\section{Tr. Albion alter}

\section{Barm 1874 Died 1925}

During the past year we have lost by death one of the small group of men to whom the foundation of this society was due and one who later became its President, Dr. Albion Walter Hewlett.

While still a medical student his intellectual ability, his industry and his power of clear thinking and accurate expression marked him as one who was destined to become an important figure in his profession.

He very early began to exhibit an interest in the fundamental processes concerned in disease, and was not content merely to observe and describe the superficial and obvious. This interest led him to the study of normal physiological processes and to apply the methods of this study to the sick.

These interests, which twenty years ago were not so common as they are now, led him and others of like mind to gravitate together, and as a result there came into being this organization of men bound together for searching out the underlying secrets of disease.

But Dr. Hewlett was also interested in men, in sick individuals, in the classification of disease, in diagnosis and in treatment. He was a skillful clinician. These qualities were the ideal ones for the successful teacher and leader, and it was not surprising therefore that he was called, while very young, to become the Professor of Medicine at one of the important universities, the University of Michigan.

While at Ann Arbor, Dr. Hewlett endeared himself to faculty and student body alike. His sincerity and the thoroughness with which he executed the trust placed in him as head of the department of medicine was an inspiration and stimulation to all, particularly to the young 
men who were fortunate enough to become intimately associated with him.

Under Dr. Hewlett's direction the Department of Medicine at Michigan continued to grow and made marked and well recognized advancement. Under his leadership valuable contributions to scientific medicine were made.

Later he was called to the home of his boyhood, to become Professor of Medicine at Leland Stanford University in California. Here he continued this valuable work as a physician, teacher and investigator.

Dr. Hewlett possessed not only an unusual intellectual equipment and ability as an investigator, teacher and physician, but also a most attractive personality. Quiet and thoughtful, and giving the impression of much reserve power and force, yet he was a most interesting and agreeable companion. All the members of the early group comprising this society were his personal friends. He was always interested in the younger members of this society and many of them were guided in their later careers by his writings and by his personal influence.

The profession of medicine has lost in Dr. Hewlett one of its ablest and most valuable members; this society has lost one of its wisest and most capable counselors.

But we have lost much more, we have all lost a sincere and true friend. It is therefore especially fitting for this society to record our admiration for Dr. Hewlett as an able scientist, our appreciation of him as a wise and skillful physician, and at the same time express the personal affection which all of us had for him as a fellow worker and a friend.

\section{PRESIDENT'S ADDRESS}

MALARIA: SOME INTERESTING OBSERVATIONS RELATIVE TO MALARIA

THAT HAVE BEEN MADE IN THE TREATMENT OF GENERAL PARALYSIS BY INOCULATION WITH MALARIA PLASMODIA

C. C. BASS

In 1917, Wagner von Jauregg (1) first reported the treatment of general paralysis by inoculation with malaria. Favorable results were obtained in six of the nine cases, three of which were later re- 
ported to be still actively at work, after four years. In 1922, he reported more than 200 cases treated by this method, over 50 of which showed remissions sufficient to permit the majority of them to resume their former occupations. His early report was followed by reports of other observers in Germany and Austria particularly, but still later in several other countries (2) (3) (4) (5) (6) (7). Reports of apparently favorable results in this disease, for which no satisfactory treatment had been available hitherto, have led to an ever increasing employment of this method until at the present time the literature contains reports of a few thousand cases. Most of the reports indicate favorable results in a proportion of cases ranging from almost none up to as high as 88 per cent (8).

On the whole, the results published are most encouraging. However, still more time and experience may be necessary to show how much allowance should be made for over enthusiasm in the use of a new and somewhat spectacular method of treatment and the difficulty of controlling the personal factor in the interpretation of the clinical state, especially in nervous and mental diseases. This applies particularly to syphilis of the nervous system in which the condition is chronic. The progress is often extremely slow; there are spontaneous improvements and the symptomatology is such that one is easily led astray. There is great danger of misinterpreting slight remissions as arrest and of attributing apparent results to treatment when they would have occurred otherwise.

Whatever therapeutic value this method of treatment may prove to have, the deliberate inoculation of hundreds and even thousands of patients with malaria under favorable conditions of control, in many particulars at least, has afforded an opportunity for observations upon certain features of malaria, far greater than had previously existed. These observations have served to emphasize certain facts in regard to malaria which were already well known but perhaps not so thoroughly appreciated and also to bring to light other facts about which much less was known. Since the observations with regard to malaria were made incidentally in most instances, when the main objective was treatment of another entirely different disease, they are probably less influenced by pre-formed ideas. My purpose is to call attention briefly to some of these points which may be called byproducts of clinical investigation of the treatment of general paralysis. 
Method of inoculation. Blood drawn from the vein of a malaria subject has been injected intramuscularly, subcutaneously or intravenously. Inoculation directly into the blood stream is somewhat more certain to infect, although both subcutaneous and intramuscular inoculations are nearly always successful. Injection of blood immediately after withdrawal gives best results, but citrated blood has been used successfully in some instances several hours after its withdrawal. In one instance (9) defibrinated blood was kept 'on ice' for 65 hours and used successfully. By keeping it on ice, it has retained its infectivity after transportation over long distances. Inoculation by the bites of infected mosquitoes has been employed, but it has been found that on the average, cases inoculated in this way, are much more difficult to cure of their infection permanently. In one instance reported by Davidson (10) of 23 mosquito infected cases, 56.5 per cent relapsed after 30 grains quinine daily for 3 days, whereas of 33 inoculated cases only 3.3 per cent relapsed. Many others have noted the infrequency of relapse of inoculated cases of malaria. One of the facts that seems to be established by these studies is that passage of malaria parasites through the sporozoite cycle in the mosquito increases their resistance in the human body.

Incubation period. The incubation period, measured by the appearance of parasites in the peripheral blood and the occurrence of fever, varies according to the mode of infection. It averages about fourteen days following infection by mosquitoes, a little longer following intramuscular or subcutaneous inoculation, and about seven days following intravenous inoculation. Clinical symptoms do not seem to be influenced by the mode of infection.

Different strains. Considerable variation in different strains of the same variety of parasite with regard to the height of the temperature has been found. For instance, Bunker and Kirby (11), using two different strains of $P$. vivax, found with one that the temperature reached $105^{\circ} \mathrm{F}$. in 23 per cent of the cases, while with the other, 86 per cent reached this temperature. Only 3 per cent of the cases inoculated with one of the strains reached $106^{\circ} \mathrm{F}$., while 64 per cent of the cases inoculated with the other one reached this height. Other effects, such as jaundice and anemia, are much more marked from certain strains than from others of the same variety of parasites. 
Immunity. Although it has been possible to infect nearly all cases, a few have been found that seem not to be susceptible. Bunker and Kirby (11) cite one patient who failed to acquire malaria after four widely separated intravenous inoculations, although other patients inoculated with the same blood at the same time developed the disease in the usual manner. A good many cases are also reported where patients who had recovered from a successful inoculation, either spontaneously or as the result of treatment, were immune and could not be infected again. Acquired immunity therefore exists, although rarely.

Spontaneous recovery. A great many cases reported have lost their infection and entirely recovered after only a few paroxysms, without any quinine or other treatment. This often occurs in the same way in naturally infected persons, as is well known to those who are familiar with malaria. In fact, in regions where malaria is very prevalent, there are a great many infected persons who have few or no symptoms, although parasites in their blood are easily demonstrated. Spontaneous recovery occurs in many of these and this is followed by more or less immunity from subsequent infection. However, we have shown (12) several years ago that whatever immunity follows spontaneous recovery is probably of short duration.

Effect of treatment. The effect of treatment in these inoculated cases of malaria under favorable conditions for observation has been impressive and is probably the most important fact from a practical standpoint that has been brought out. The experiences reported emphasize the effectiveness of quinine in the treatment of malaria and indicate that the large amount often given to malaria patients is unnecessary. The treatment used by the different observers has varied considerably, ranging from 5 to 30 grains quinine (usually by mouth) daily for periods of from three days to two weeks and occasionally, but rarely, longer. Among the three or four thousand cases reported, the clinical attacks were promptly controlled, usually within 24 hours and always within two or three days. This is in line with the experience of many students of malaria to the effect that attacks of this disease are always promptly controlled by the use of moderate doses of quinine.

Clinical investigation or clinical research, the encouragement and 
promotion of which is the object of this Society, has, in this instance, not only furnished information relative to what it now seems may be a valuable therapeutic agent for general paralysis, but at the same time it has shed new light on the entirely unrelated disease, malaria, and has impressively emphasized certain previously known facts relative to this disease.

\section{BIBLIOGRAPHY}

1. Wagner von Jauregg, Julius: Psychiat.-Neurol. Schnschr., 1918, xx, 132; 1919, xxi, 251. Ueber die Einwirhung der Malaria auf die progressive Paralyse.

2. Mühlens: Arch. f. Schiffs u. Trop. Hyg., 1920, xxiv, 173.

3. Gertsmann: Zeit. f. d. ges. neurol. Psychiat., 1912, lxxiv.

4. Kirschbaum: Zeit f. d. gesamte Neuro-Psychiatrie, 1922, lxxv.

5. Hauptmann: Zeit. f. Hyg. u. Infektionskr., 1921, xci, 1.

6. Riddel and Stewart: Jour. Neurol. and Psychopath., 1923, iii, 12.

7. Delgado: Jour. Nerv. and Mental Dis., 1922, lv, 376.

8. Weigandt: Muench. med. Woch., 1920; Zeit. f. d. ges. Neur. u. Psych., 1922, lxxxi.

9. Clark, R. M.: Brit. Med. Jour., 1925, i, 600. Treatment of General Paralysis by Malaria.

10. Davidson, T. Wishart: Brit. Med. Jour., 1925, i, 452. Treatment of General Paralysis by Malaria.

11. Bunker, Henry A., Jr., and Kirby, George H.: Jour. Amer. Med. Ass., 1925, Ixxxiv, 563. Treatment of General Paralysis by Inoculation with Malaria.

The Relative Importance of the Systolic and Diastolic Blood Pressure in Maintaining the Coronary Circulation. By FRED M. SMITH and (by invitation) G. H. MILLER and V. C. GraBer, Iowa City.

The object of the investigation was to determine the effect of specific changes in the systolic and diastolic blood pressure on the coronary circulation.

Dogs were employed. They were anaesthesized and the blood pressure from the carotid artery was registered on the kymograph by a Straub membrane manometer. The chest was opened and a Morawitz-Zahn cannula was introduced into the coronary sinus. The blood was prevented from coagulating by heparin. The blood from the coronary sinus was collected in a $50 \mathrm{cc}$. graduate, and the amounts were registered on the kymograph. The blood was maintained at a constant temperature and reintroduced into the femoral vein. Changes in the systolic and diastolic pressures were induced by constricting the thoracic aorta, and by experimentally produced arterio-venous aneurysm and aortic regurgitation.

When the thoracic aorta was constricted by an adjustable clamp, it was possible, within certain limits, to increase the diastolic pressure without altering the systolic pressure. The coronary flow was increased in proportion to the elevation of 
diastolic pressure. When the arterio-venous aneurysm was employed, the diastolic pressure was reduced and the systolic slightly elevated and the rate of coronary flow was decreased in spite of the accompanying elevation of the systolic pressure. In those instances in which the diastolic pressure was reduced by puncturing the aortic valves, a similar, but even more striking reduction in coronary flow was obtained.

The Relationship of the Hydrogen Ion to the Genesis of the Cardiac Rhythm. By Edward P. Carter and (by invitation) E. Cowles ANDrus, Baltimore.

Changes in the $\mathrm{H}$ ion concentration of the fluid bathing the heart is shown to affect the origin and spread of the excitatory process as follows:

1. A change from $\mathrm{pH} 7.4$ to $\mathrm{pH} 7.0$ causes slowing of the rhythm, lengthens the $\mathrm{P}-\mathrm{R}$ interval and depresses intra-auricular conduction.

2. Raising the $\mathrm{pH}$ from 7.4 to 7.8 results in a quickening of the rhythm and an increase in the rate of conduction.

3. Vagomimetic substances are less effective at $\mathrm{pH} 7.8$ and more effective at $\mathrm{pH}$ 7.0. Conversely the action of sympathomimetic drugs is enhanced at $\mathrm{pH} 7.8$ and depressed at $\mathrm{pH}$ 7.0.

4. Studies of the results of altering the $\mathrm{Ca}$ concentration at constant $\mathrm{pH}$ indicate that the effects of $\mathrm{pH}$ changes cannot be due to the changes in the ionization of the $\mathrm{Ca}$ in solution.

5. There is evidence that carbonic acid or its ions may exert a "specific effect" upon the rhythm. An increase in the $\mathrm{H}_{2} \mathrm{CO}_{3}$ content of the perfusate brought about without change in the $\mathrm{pH}$ of the solution causes an increase in the rate of spontaneous development and propagation of the excitatory process. In a certain proportion of experiments, however, this effect comes on more slowly than the effects resulting from a change from a normal to a more alkaline perfusate and it tends also to pass off more gradually after return to the solution containing little or no carbonic acid. The ability of carbonic acid or its ions to penetrate the cell and to raise the $\mathrm{cH}$ of its contents is suggested as an explanation of this "specific effect."

Upon the basis of these results the authors suggest that the rate of origin and propagation of the excitatory process in the heart is dependent upon the difference in $\mathrm{H}$ ion concentration within and without the cardiac cells.

The Effect of Irregularity of the Rhythm of the Heart on the Blood Flow in Dogs.

By H. J. Stewart, J. H. Crawford and A. B. Hastings (by invitation) and

A. E. CoHN.

Evidence has been obtained of the rate of blood flow in unanesthetized dogs during experimental auricular fibrillation. Two insulated wire electrodes were sutured to the right auricle under aseptic technique 24 hours preceding the experiment. Auricular fibrillation was induced by faradic stimulation of the auricles and rapid regular rhythms by single induced shocks at known regular rates. Electrocardiograms were taken before, during and after stimulation. Arterial 
blood was obtained from a femoral artery. Samples of mixed venous blood were obtained by a technique devised by Stewart. Oxygen contents and capacities of the arterial and mixed venous blood were determined by means of Van Slyke's manometric method before, during and after the induced rhythms.

Summary. 1. During auricular fibrillation the oxygen saturation of the arterial blood was unchanged; during regular tachycardia it was usually unchanged but was occasionally decreased 4 to 7 per cent.

2. Calculations of the relative changes in blood flow during the period of auricular fibrillation indicate decreases varying from 20 to 62 per cent of the normal.

3. The blood flow during regular tachycardia was unchanged in 11 observations in 7 dogs and decreased in 5 observations in 3 dogs.

4. In 5 experiments the same or comparable ventricular rates were obtained during auricular fibrillation and during regular tachycardia in the same dog. The blood flow was decreased in auricular fibrillation but was decreased in the regular tachycardia in only one observation.

Factors such as ventricular rate and pulse deficit concerned in determining when this difference in the circulation rate between auricular fibrillation and regular tachycardia occurs are now being analysed.

The Velocity of Blood Flow in Health and Disease. By Herrmann Blumgart and (by invitation) Soma Werss, Boston.

The velocity of blood flow has been determined in over one hundred and fifty patients between the ages of eighteen and seventy-five. Active deposit of radium was injected into the cubital vein of one arm. When the active deposit reached the cubital arterial vessels of the other arm, the beta particles and gamma rays emerged through the tissues of the arm and were automatically registered by an appropriate detecting device. No withdrawal of blood was necessary. No toxic effects have been noted.

In fifty normal individuals in whom the venous pressure and vital capacity were normal, the time from elbow to elbow has ranged from fifteen to twenty-four seconds. The average time was eighteen seconds and only nine individuals exceeded twenty seconds. In a given individual, successive determinations with varied dosages usually checked within two seconds, in a few instances within three seconds. Circulation times above twenty-four seconds were always associated with pathological conditions in the circulatory system. In twelve patients with auricular fibrillation the time averaged forty-four seconds with a range of twenty-eight to sixty. In four patients when sinus rhythm was induced by quinidine the time was shortened, on the average, eight seconds with a range of from two to sixteen seconds.

In emphysematous patients in whom the vital capacity was conspicuously reduced, the time was normal in some and prolonged in others, according to the degree of myocardial involvement.

Observations in other diseases were made and further studies are in progress. 
Studies of Vital Capacity in the Negro Race. By Wrison G. SmILlIE and (by invitation) Donald C. Augustine, New York.

In the course of an investigation to determine the effect of varying intensities of hookworm infestation upon the rate of growth and development a group of about two thousand school children in south Alabama was studied. Negro children were observed as well as whites. The standard measurements for normal children developed by the U. S. P. H. Service were used; i.e., standing height, sitting height, weight, certain strength tests, vital capacity and hemoglobin.

It was discovered that normal negro children have a markedly lower vital capacity than normal white children of the same age, sex and weight. The standing height curve of growth of the two races was found to be quite similar but the trunk length or sitting height of the white children was found to be greater than that of negro children.

In order to determine whether or not the strikingly low vital capacity observed in negroes was limited to children only, a group of about two hundred strong, healthy, white and negro adults was studied. These men were all prisoners at a large convict logging camp. The results showed that adult negroes as well as negro children have a vital capacity markedly lower than whites.

\section{Distribution of Jaundice in Circulatory Failure. By Jonathan Meakins, Mon- treal.}

The cases are divisible into two classes, (a) Biliary pigmentation generalized, (b) Biliary pigmentation localized to the upper part of the body, head and arms. Six cases were studied. All had severe circulatory failure and all had tricuspid insufficiency. Four cases died, with autopsy on three. One case of generalized pigmentation, which developed under observation, had acute tricuspid insufficiency with jaundice before anasarca became pronounced. The blood serum and oedema fluid of this patient were both bile stained. In five patients with localized pigmentation severe anasarca was present before serious tricuspid insufficiency and jaundice developed. Biliary pigmentation was confined to areas where oedema or ascites was not present. If hands and fore-arms were oedematous jaundice did not occur in these areas. One case extensively studied developed jaundice under observation. The blood serum was always bile stained and gave a strong direct and indirect van den Bergh reaction. The oedema fluid and the ascitic fluid was not bile stained and van den Bergh reactions on them were negative. The case studied throughout revealed a spread of the jaundice as the oedema fluid was drained away by repeated skin punctures. The questions arise whether the oedema fluid actually is not in diffusion equilibrium with the blood plasma in cardiac oedema or whether only colloids with large molecules do not diffuse. Crystaloids, urea, uric acid, creatinine and sugar are in fair equilibrium. Proteins, haemoglobin and bile pigments are much diminished or absent. Bile salts may be in equilibrium as the surface tension is practically the same in both fluids. The histopathology of the liver is distinctive and important. 
Studies in Obstructive Jaundice By C. H. GreENe and (by invitation) M. Aldrich and A. M. SNELL, Rochester, Minn.

Obstructive jaundice was produced experimentally in dogs by ligation of the common bile duct. Cholecystectomy was also done in some animals. The initial response after obstruction depends on the presence or absence of the gall bladder. Cholecystectomized animals show a rapid increase in biliary constituents in the blood. Jaundice is marked within twenty-four hours. When the gall bladder is intact the increase in biliary constituents in the blood is delayed and jaundice may not develop for forty-eight to seventy-two hours. This effect can be shown with bilirubin, bile salts, and dyes (phenoltetrachlorphthalein and brom-sulphalein). A maximal degree of bile retention was observed at the end of the first week, thereafter there was a gradual readjustment towards normal. The serum bilirubin tended to decrease to a level of 2 to $3 \mathrm{mg}$. even though obstruction was maintained. The bile salts varied but also tended towards normal. Dye retention persisted in these dogs, though its degree sometimes became less.

There was a rapid return towards normal with the relief of the obstruction when the latter was of short duration. After long continued obstruction recovery was greatly delayed: Slight bilirubinemia, with marked increase in bile salts and dye retention were observed in one animal for several months after cholecystgastrostomy. Ascites and the late clinical picture of obstructive biliary cirrhosis were observed in one dog with complete biliary obstruction for 112 days.

Alterations in Liver Function as an Index of Toxemia in Pneumococcus Lobar Pneumonia. By Benedict R. Harris (by invitation of Francis G. Blake), New Haven, Conn.

In order to determine the effect of pneumococcus lobar pneumonia on hepatic function, dye elimination tests were done on twenty-two cases, of which 9 were due to pneumococcus Type I, 3 to pneumococcus Type II, 5 to pneumococcus Type III, and 5 to pneumococcus Type IV. Impairment of liver function was found to occur consistently, the degree increasing as the disease progressed. No definite relationship between the severity of the infection (as measured by outcome), and the degree of liver impairment was found. Following recovery liver function returned rapidly to normal, indicating no permanent injury.

Impairment of function in the Type III pneumonias with 80 per cent mortality was conspicuously less than in the Types I, II, and IV, with 23 per cent mortality.

\section{An Isolated Case of Epidemic Influenzal Pneumonia. By Channing Frothing- HAM, Boston.}

The terms, influenza and influenzal pneumonia, are quite loosely used by the medical profession and are applied to a variety of diseases. There is no way at the present time to separate febrile upsets of unknown cause which involve the upper respiratory tract and the epidemic disease which swept through the country in 1918-19, unless the epidemic disease reaches the lungs. It is possible by a study of the lung tissue at autopsy to pick out definite lesions which are peculiar to 
this epidemic disease, even when complicating organisms add to the pulmonary kesion.

Isolated cases of the epidemic disease that have been proven at autopsy are so rare that it seems worth while to report these cases with the hope that as time goes on it will be possible to establish their identity during life even without the complication of pneumonia. Apparently if the epidemic disease invades the lung it is possible to make a diagnosis by the peculiarity of the $x$-ray findings. This case was suspected by the $x$-ray examination during life and confirmed by the examination of the lungs at autopsy to be a case of the epidemic disease with pulmonary complications.

Changes in Serum Freezing Point and in the Concentration of Serum Electrolytes during Lobar Pneumonia. By F. WILLIAM SUNDERMan and J. G. CaMack (by invitation) and J. H. Ausrrs, Philadelphia.

The changes in electrolyte and non-electrolyte concentrations in the serum in twenty-two cases of lobar pneumonia were followed through the febrile and afebrile periods by means of freezing point, conductivity, and refractometric measurements, supplemented with total base, chloride and $\mathrm{CO}_{2}$ analyses, and with certain non-electrolyte determinations. Whenever possible, bleedings were made at intervals of two or three days on each patient before and after the crisis.

During the active infection there was a decrease in the concentration of electrolytes in the serum and a proportional decrease in the freezing point depression. After the crisis the electrolytes returned to the normal range, the total electrolytes faster than the chlorides; whereas, the freezing point depression was increased above the normal limits. This increase in freezing point depression at the time of the crisis must be due to abnormally higher concentration of non-electrolytes, the precise nature of which was not determined.

A Study of Red Blood Cell Permeability. By J. P. Peters and (by invitation) A. J. Eisenman, and A. M. Wakeman, New Haven, Conn.

Human blood was brought into equilibrium with $40 \mathrm{~mm} . \mathrm{CO}_{2}$ in air at $38^{\circ} \mathrm{C}$. Each sample of blood was divided into three parts. The first wasanalysed directly. To the second and third were added equimolar amounts of a sodium and of a potassium salt. Chloride and bicarbonate were used. Cell volume and serum protein, $\mathrm{CO}_{2}$ content and $\mathrm{Cl}$ concentration in the whole blood and the serum, and serum total base, were determined.

Eight experiments were done, all of which showed similar changes. The total base found in the serum to which the salt had been added agreed within $2 \mathrm{mM}$. with that calculated assuming no shift of base across the membrane. The variation was not consistently above or below the calculated and it was within the limits of error for the method. There was a transfer of $\mathrm{Cl}$ and $\mathrm{CO}_{2}$ across the membrane. Whether equimolar concentration of $\mathrm{Na}$ or $\mathrm{K}$ salt was added, the changes in the distribution of water, $\mathrm{Cl}$ and $\mathrm{CO}_{2}$ were quantitatively identical in 
the analyses. Therefore, within the limits of these experiments, no evidence of any change in the distribution of base between cell and serum was found.

The Carbon Dioxide Equilibrium in Alvolar Air and Arterial Blood during Exercise. By A. V. Bock and (by invitation) D. B. Dill, J. S. Lawrence and L. M. HURXTHAL, Boston.

The validity of samples of alveolar $\mathrm{CO}_{2}$ obtained by the Haldane-Priestley method during exercise has long been questioned. Data are presented showing that by means of a slight modification of the method as originally described, a close agreement between the tension of $\mathrm{CO}_{2}$ in the alveoli and that of arterial blood can be demonstrated. This fact bears an important relationship to practically all methods now employed for the determination of the total blood flow.

The Influence of Anti-rheumatic Drugs upon the Arthritis of Serum Disease. By

C. L. Derick and Charles H. Hitchcock (by invitation) and Homer F. Swift, New York.

As a result of bed-side observation, and from the character of the joint fluid of patients with serum disease it seemed that the "arthralgia" might be inflammatory in nature. Attempts were made, therefore, to determine whether these symptoms might be influenced by anti-rheumatic drugs. Previous observations had shown that little effect was discernible when the drugs were administered after the onset of "arthralgia;" hence in these series of cases the drugs were started shortly after the time the serum was discontinued, and were given for from 10 to 14 days. In one series the patients were treated with neocinchophen, in another with aspirin. No difference in the action of these two drugs could be detected. While the urticaria was unaffected, the amount of arthritis in these patients was markedly diminished. The rate and intensity of precipitin formation against horse serum and the persistence of horse serum in the blood of these patients was studied and compared with similar observations made by Longcope and Rackemann, and MacKenzie and Leake. As a rule the antibody in these drug-treated patients was much lower in concentration, and the antigen (horse serum) persisted longer than in the patients reported by the authors above mentioned. It is suggested, therefore, that the arthritis may depend upon the development of a certain concentration of antibody by the patient, and that the depression of antibody formation, the result of the drug administered, may be the cause of the decreased amount of arthritis.

Experimental Nephritis Produced by X-ray. By James P. O'Hare and (by invitation) Hugo O. Altnow, Thomas D. Christian, Jr., and Abner Calmoun, Boston.

In the first groun of rabbits incision was made over the right kidney which was drawn up into the wound and exposed once to direct action of $x$-ray. In different rabbits of this group, $\mathrm{x}$-ray exposure varied in degree. The animals were killed 
at intervals, varying from one day to four and a half months. Minor changes were noted in the rabbits exposed to smaller dosage and sacrificed early. In rabbits exposed to the larger dosage and allowed to live for three months or more, there was marked sclerosis of the kidney, reducing the organ in some cases to onehalf the size of the control left kidney.

A second series was treated in a similar way except that both kidneys were exposed to the $\mathrm{x}$-ray, the second kidney after the animal had fully recovered from the operation on the first kidney.

The Action of Parathyroid upon Calcium and Lead in the Bones. By Donald HunTER (by invitation) and J. C. AUB, Boston.

Patients with lead poisoning were given a calcium deficient diet containing 110 mgms. of Ca daily, so that they excreted more calcium than they ingested. The total output of calcium and lead in urine and feces was estimated.

Collip's parathyroid extract was then injected twice daily. In five out of six patients increasing doses of the extract caused a gradual increase of the blood calcium to 13 or $14 \mathrm{mgms}$. per $100 \mathrm{cc}$. The excretion of both calcium and lead then rose markedly. The lead excretion rose on several occasions to over 2.5 mgms. a day, a level nearly twice as high as that obtained in our previous methods of treatment. No untoward symptoms were observed.

The lead which was excreted in these observations had been stored almost entirely in the bones. The excess of calcium must have come very largely from the bones. Therefore these observations indicate that parathyroid may cause a mobilization and excretion of inorganic salts from the bones. They also contribute to the evidence that lead and calcium run parallel in their reactions in the body. This method offers a very effective but somewhat unsafe treatment for the elimination of lead.

Mechanism of the Action of Iodides on the Nitrogen Metabolism. By G.P.GraBFIELD and (by invitation) C. GRAY and B. FlowER, Boston.

It has been previously shown that the administration of iodides to human subjects causes an increase in the nitrogen excretion. Hesse has shown that a similar reaction occurs in dogs.

The present work was done on dogs maintained in positive nitrogen balance on a diet containing 2.8 grams of nitrogen and a total of $660 \mathrm{cc}$. of fluid. After the nitrogen excretion had become constant, sodium iodide was administered subcutaneously in doses of $\mathbf{0 . 4}$ grams per kilo per day for three days. During the period of drug administration the total nitrogen of the urine rose on the average 23 per cent a day, returning to the previous level after the administration was stopped. The total sulphur determined by method of Fiske remained unchanged.

After thyroldectomy the same experiment was carried out but the injection of iodides in the absence of the thyroid failed to cause an increase in the nitrogen excretion. 
It is concluded, therefore, that this increase in nitrogen excretion is due not to the breaking down of the body protein but to an excretion of "deposit nitrogen" containing no sulphur and that the presence of the thyroid is necessary for this action of iodides.

Blood Volume in Myxedema. By W. O. Thompson (by invitation of J. H. Means), Boston.

Nine patients with myxedema averaged 22 per cent more total plasma, 28 per cent more plasma per kilo and 25 per cent more plasma per square meter of surface area when fed thyroid than when myxedematous.

From observed and calculated changes in total blood volume, it is estimated that thyroid feeding in myxedema quite commonly produces increases of 25 per cent in total volume of circulating blood.

The plasma decrease on omitting the thyroid and the increase on restarting it occur much more rapidly than do the corresponding changes in the volume of cells. Plasma reduction is usually well marked within ten days after omitting thyroid.

A parallelism exists between basal metabolic rate and plasma volume in myxedema.

Once the basal metabolism has been restored to normal further increases in the dose of thyroid up to three times the maintenance dose produce only slightly greater increases in plasma volume and basal metabolism than does the maintenance dose.

The plasma volume changes in myxedema are contrasted with those in cardiac edema in which plasma volume increases with increasing edema. Other differences between the two are noted.

Observations on Polycythemia Vera (Erythremia) during Treatment by Phenylhydrazin. By G. E. Brown and H. Z. GIFFIN, Rochester, Minn.

Seven patients with polycythemia vera have been treated by phenylhydrazin hydrochloride. In addition to the clinical data the observations on these cases include among other things estimations of the blood volume and viscosity, studies in blood chemistry, functional tests on the liver and the kidneys, electrocardiographic studies, estimations of heat production, and a general study of the circulatory system under stress. An effort is made to arrive at some conclusions concerning the toxicity of phenylhydrazin when administered by mouth and the significance of the jaundice and of the thrombosis which have occurred during treatment.

An Investigation Relative to B. Welchii Infection of the Intestinal Tract as the Etiological Factor in Pernicious Anemia. By RoBERT N. NyE, Boston.

In confirmation of the work of others a very great increase in B. welchii spores was found in stools from cases of pernicious anemia. A similar increase was found 
in stools from cases with achylia gastrica without pernicious anemia. Crude estimates of vegetative forms showed at least no more in pernicious anemia stools than in those from normal individuals.

Contrary to general belief, it seems probable that the reaction of the normal small intestine, at least as far as the mid portion, possibly as far as the cecum, is decidedly on the acid side of neutrality, due chiefly to the presence of normal gastric juice. A neutral or slightly alkaline reaction is necessary for active sporulation of $\mathrm{B}$. welchii, hence spore production in at least two thirds of the normal gastro-intestinal tract is at a minimum. However, in conditions of achylia gastrica such inhibitory influences are lacking and a marked increase in spores without increase in active or vegetative forms results. Therefore, the spore increase in pernicious anemia is probably secondary to the achylia gastrica rather than indicative that chronic intestinal infection with $B$. welchii is the etiological factor in pernicious anemia.

Studies on the Relation of Monilia to Pernicious Anemia. By G. O. Broun and (by invitation) CECrEE JACOBSON and O. GARCIA, St. Louis.

Rabbits and guinea pigs were inoculated with monilia isolated from the stools of active cases of pernicious anemia. Intravenous, subcutaneous and intraperitoneal injections of monilia, while causing slight decreases in the red count and hemoglobin of some animals, in no instance produced a picture approximating pernicious anemia in man. After inoculation, agglutinins and complement fixing antibodies for monilia appeared in the blood.

Animals fed large numbers of monilia by mouth, over long periods of time, developed no anemia. The organisms could be recovered from the stools while the feedings were in progress, but quickly disappeared if the feedings were discontinued.

The sera of a number of cases of pernicious anemia from which monilia have been isolated showed positive complement fixation tests with antigen prepared from monilia. The reaction is specific, in that sera giving positive Wassermann and positive tuberculosis complement fixation tests give negative results with the monilia antigen. Monilia can also be found in other conditions than pernicious anemia. Sera of a number of such cases have given positive complement fixation tests with antigen prepared from monilia isolated from a case of pernicious anemia.

Studies on Gastric Anacidity. By C. S. KeEFer (by invitation) and A. L. BloomFIELD, Baltimore.

During the course of some studies on gastric secretion a method was devised by which the volume of gastric secretion and discharge could be measured quantitatively. Applying this method to cases of gastric anacidity we have been able to obtain some information regarding the volume of secretion in such cases.

We divided the cases into three groups and compared them according to the 
average volume of gastric juice secreted in 10 minute periods, with the following results:

Group A. Control Series.

Group B. Anacidity cases without gastric disease.

Group C. Anacidity cases with gastric disease.

Percentage of cases with volumes of 10 to $40 \mathrm{cc}$.:

Group $\mathrm{A}=89$ per cent

Group B $=93$ per cent

Group $\mathrm{C}=40$ per cent

Percentage of cases with volumes of less than $10 \mathrm{cc}$.:

Group $\mathrm{A}=3.5$ per cent

Group $B=7.0$ per cent

Group $\mathrm{C}=60.0$ per cent

It is apparent from these results that while the cases of anacidity without gastric disease have practically the same volume of secretion as the normal controls, the cases of anacidity with gastric disease have a very much smaller volume of secretion. In other words in any case of gastric anacidity in which the volume secretion is below $10 \mathrm{cc}$. (average of several 10 minute periods) one is probably dealing with organic gastric disease, whereas, if volume of secretion if over $10 \mathrm{cc}$. it is more likely to be a functional anacidity.

Preferential Utilization of Carbohydrates in Diabetes. By IALter R. Campbell and (by invitation) J. Markowitz, Toronto.

In the milder cases of diabetes there has appeared to be some grounds for the belief that certain carbohydrates are more easily utilized by the diabetic organism than other carbohydrates. Information on this point would be of great value in treatment but the possibilities of stimulation of insulin production by the carbohydrate or by exercise, of storage of the carbohydrate as fat, as blood and tissue sugar, or as glycogen, and of non-absorption from the alimentary tract, have seldom been given sufficient attention in arriving at a conclusion. By a new type of experiment an attempt has been made to throw some light on this problem. On a weighed diet, consisting of meat, raw pancreas and glucose, totally depancreatized dogs receiving insulin twice daily with meals and kept in nitrogenous equilibrium excrete a fairly constant amount of sugar daily. After a control period the carbohydrate to be tested is substituted for the glucose in the diet. After the test period a further control period using glucose is carried out. The urine is carefully collected and analysed for sugar, nitrogen and phosphates. While such a method has certain drawbacks it also has the advantages of adequate control of food intake, insulin dosage and other conditions of the experiment which are not so satisfactorily carried out on diabetic patients. The substances chosen for test were levulose, inulin, glycerin, and a triose-dihydroxyacetone. If a limited quantity of insulin permits a greater amount of the sugar to be utilized by the 
animal the urinary excretion of glucose will decrease by this amount. Within the limits of error of such a method no preferential utilization of these substances was demonstrated.

Diabetic Children's Diets in Different Clinics, Calculated from 532 Reports. By $\mathbf{H}$. Gray, Santa Barbara.

Stimulated by the tabulation by Holt and Fales of normal children's diets in grams per kilogram of body weight, a similar tabulation was undertaken for diabetic children. The great courtesy of a number of prominent students of diabetes in furnishing details on their patients, with a smaller amount from the literature of similar data at the time of a patient's discharge, has made possible this survey. For each investigator averages have been made, and the differences between these and the grand average have been examined by biometric technic to determine which differences are statistically significant. It is believed that this method reveals more precisely than is now known the diet distribution most favored in the years since insulin became available, and likewise clarifies such trends as diverge sufficiently from the average to demand discussion.

Metabolism during Fasting in the Human Subject. By William G. Lennox, Boston.

Daily measurements of oxygen consumption, of nitrogen excretion, and of bicarbonate, sugar and non-protein nitrogen in the blood plasma were made during five fasting periods lasting from 6 to 15 days. Before the fasts, subjects were on a much lower protein intake than the subjects of most of the fasts reported in the literature. The amount of oxygen consumed, per kilogram of body weight, was increased thoughout fasting in each case. Such increase, except under abnormal conditions of fuid intake, ran roughly parallel to nitrogen excretion.

Exaggerated Response of Sensitized Smooth Muscle to Mixtures of Sensitized Tissue and Homologous Antigen. By Harry L. AleXander, and (by invitation) WM. G. BECKE and J. H. Holmes, St. Louis.

Guinea pigs were sensitized by intraperitoneal injections of antigenic substances of varying nitrogen content. Both uterine horns were suspended, each in a separate Dale apparatus. In one Dale bath, a measured amount of homologous antigen was placed, and the amplitude of muscle contraction recorded. To the other, the supernatant fluid of a mixture containing the same measured amount of antigen ground with the perfused lungs of the same animal was added. The resulting muscle contraction was frequently greater than when antigen unmixed with tissue was used.

A similar exaggerated muscle response was occasionally obtained with mixtures of sensitized liver, uterine or kidney tissue and antigen. This exaggerated response was not constant. To account for this, two possibilities are considered.

1. In the mixtures used, there is not always a correct proportion between the 
amount of antigen and tissue. This was not demonstrated because the conditions of such an experiment could not be kept constant.

2. The tissue factor in the mixtures that causes exaggerated smooth muscle response may be unevenly distributed among various organs. This is indicated by the discrepancies in the curves obtained by comparing the response to mixtures of lung tissue and antigen with those of other tissues and antigen under similar conditions.

Observations on the Nature of "Heart-Burn." By CHeSTER M. JONES and (by invitation) WYMAN RichaRdson, Boston.

Twenty-nine normal subjects have been studied for the production of "heartburn" by distension of the oesophagus at different levels with a balloon, the location of which was determined by fluoroscope. Nineteen subjects noticed a hot burning sensation during distension of the lower third of the oesophagus. The remainder complained of fullness or pressure. Distension of the middle or upper third of the oesophagus caused a sensation of pressure in twenty-five cases and burning in only four. Introduction, through a tube, of small quantities of acid, alkali, cold water, gastric contents, or barium suspension into the lower third of the oesophagus caused "heart-burn" in two-thirds of those in whom it was attempted. Fluoroscopic examination of the barium column showed the presence of spasm at the level at which the liquid had been introduced, with reverse peristalsis above this level. The spasm and reverse peristalsis persisted as long as the "heartburn" lasted.

In the above observations simple local distension seemed to be the cause of the sensations. It is probable that "heart-burn" is analagous to referred pain and hunger contractions, and is a reflex disturbance of lower oesophageal tone, usually with abnormal persistalsis, occasioned either by local pathology or by functional or organic pathology elsewhere in the body.

Oxygen Poisoning. By C. A. L. BINGer and (by invitation) J. M. FAulkner and R. L. Moore, New York.

The present day, rather widespread, therapeutic use of oxygen makes it important for us to have more exact knowledge about its deleterious effects. The history of this subject dates back to the work of Paul Bert, who showed that high atmospheric pressures of oxygen produced convulsions in animals. Bert did not observe the characteristic pulmonary changes because his animals died before these changes occurred. Lorrain Smith was the first to describe the lesions in the lungs. His work has since been confirmed by others, notably by Karsner, who made a thorough study of the morbid anatomy of rabbits exposed to high partial pressures of oxygen.

It was our purpose to inquire into the nature of the pulmonary lesions, to study the limits of oxygen concentration at which they developed and to make certain that there was no secondary bacterial invasion. We were interested, too, in ob- 
serving the effects of high concentrations of oxygen on other species, and in learning, if possible, the cause of death.

We have found that mice, guinea pigs, rabbits and dogs all succumb to the effects of oxygen in concentrations of 80 per cent of one atmosphere or over in about 5 days. These animals at first show no untoward effects. They gradually, however, stop eating, lose weight, become dyspneic and cyanotic and then die. When they are in the advanced stage of dyspnea, removal from the high concentrations of oxygen brings on immediate death. Examination of their blood shows a marked anoxemia.

Apparently the pulmonary lesion is such as to interfere with the diffusion of oxygen and the same high concentrations which eventually kill the animals temporarily keep them alive. The characteristic pulmonary lesion is capillary dilatation and an hemorrhagic edema.

We are at present investigating the effect of similar concentrations of oxygen on amphibia and reptiles. Apparently the winter frog is able to survive high concentrations, though whether there is any statistical difference in his viability in oxygen as compared with air, we are not yet certain. We are at present engaged in the study of the effect of oxygen on the capillaries.

The Nephrotoxic Action of Ingested Cystine. By A. C. CuRTIs (by invitation) and

L. H. NewBURGH, Ann Arbor, Mich.

It has been shown by Newburgh and Marsh that the intravenous injection of cystine into dogs causes marked injury of the kidney. H. B. Lewis has shown that the kidneys of rabbits are injured by the introduction into the stomach of single large doses of cystine.

The former experiments are open to the criticism that the cystine entered the organism by an abnormal path. In the latter experiments, the introduction of a single large dose into the stomach of a fasting animal permits much more rapid absorption than would occur if an excess of cystine were taken in the usual way.

Our experiments deal with the effect of cystine when ingested as a constituent of the diet by the rat, and show that:

1. Large doses cause a hemorrhagic nephropathy and death within a few days.

2. Doses only several times the minimal requirement cause moderate renal injury in the course of months.

3. The addition of cystine to diets in themselves too low in cystine to permit growth will, in small doses cause growth; in moderate doses inhibit growth; and in large doses produce actual loss of weight.

The Respiratory and Carbohyrate Metabolism of Dihydroxyacetone in Normal and Diabetic Individuals. By EDWARD H. MAson, Montreal.

Normals: Comparable respiratory and blood sugar time curves after the ingestion of like doses, 25 or $\mathbf{5 0}$ grams, of glucose or dihydroxyacetone, in ten experiments on five cases have shown the following results: 
1. The average maximum increment increase of the non-protein respiratory quotient after glucose was 0.026 , while after dihydroxyacetone it was 0.271 .

2. The average maximum increase of heat production after glucose was 10.6 per cent as against 16.1 per cent for dihydroxyacetone.

3. The blood sugar showed a lesser increment increase after the dihydroxyacetone.

Diabetes: Fifteen similar experiments on eight cases gave the following results:

1. The average maximum increment increase of the non-protein respiratory quotient after glucose was 0.048 , while after dihydroxyacetone it was 0.138 . .

2. The average maximum increase of heat production after glucose was 11.2 per cent, while after dihydroxyacetone it was 19.4 per cent.

3 . The blood sugar showed a lesser increment increase after the dihydroxyacetone.

Studies in Albuminuria. By HILdINg Berglund and (by invitation) WALTER SCrIver, Minneapolis, Minn.

Factors influencing the albumin output through diseased kidneys have been studied in cases of acute and chronic nephrosis as well as in animal experiments. Both serum-albumin and serum-globulin usually escape into the urine. Albuminuria without globulinuria may occur but is evidently rare. A high albumin to globulin ratio in the urine signifies a lesser degree of abnormal permeability than a low ratio. The urinary albumin to globulin ratio is always higher than 1 . The urinary ratio is independent of the corresponding ratio of the plasma.

On a high protein diet the total protein output through the kidneys may become increased as much as three times above the output during a previous or following period of low protein diet. Increased elimination of urea after urea feeding has no similar effect on the amount of urinary protein.

This phenomenon does not indicate increased kidney damage. In fact, it indicates a greater amount of protein circulating through the kidneys. This statement is based upon observations on the concentration of the plasma proteins. The protein synthesis after high protein feeding is a rapid process. In three days the plasma proteins may increase 30 per cent.

A Study of Supravital and Fixed Stained Preparations of Cells Obtained by Puncturing Diseased Lymphnodes in Man. By ClaudE E. ForkNER (by invitation) and George R. Minot, Boston.

Biopsy for diagnostic and prognostic information has certain disadvantages. Puncture of lymphnodes causes less discomfort to the patient and useful knowledge can be obtained rapidly by studying in supravital and fixed stained preparations cells so obtained. Material can be withdrawn on the barbed edges of a dental broach passing through the lumen of a needle, which has perforated the capsule of a lymphnode. The supravital technic offers a new method of approach to the study of the cell content of diseased lymphnodes. The conditions studied include 
various types of lymphoblastoma, metastatic carcinoma, tuberculosis and simple hyperplasia. Carcinoma is distinguished easily. The significance of the cell pictures obtained in lymphoblastoma can be evaluated after collecting much data.

The Experimental Production of Lung Abscess. By S. A. Schlueter, and I. F. WeIDLEIN (by invitation) and E. C. CuTLER, Cleveland, Ohio.

A successful method for the experimental production of lung abscess has been devised in this Laboratory. The procedure consists in liberating into the jugular vein an artificial, infected embolus which is carried in the venous blood stream to the right heart and thence by way of the pulmonary artery to the lung.

The infected embolus is prepared as follows: A small segment of femoral vein, 6 to $8 \mathrm{~mm}$. in length, is excised. One end is tied with silk. The other end is held open by three silk sutures, and various bacterial emulsions are transferred into the lumen with a platinum loop, together with a bit of paraffinized lead. A few drops of blood are then purposely added to serve as a temporary culture medium, and the material is encased with a second silk ligature about the open end. The embolus is then placed in a glass cannula, and the latter connected to a syringe filled with saline. An opening is made into the jugular vein, the cannula inserted and the embolus forced into the circulation. The lead filing serves to localize roentgenographically the final resting place of the embolus.

Success depends only on the type of organisms employed. With the proper bacteria, the lesion has resulted in 100 per cent of the experiments.

Guanidine and Hypertension: Clinical and Laboratory Studies. By C. P. HowARD and (by invitation) I. M. RABINOwITCH, Montreal.

The determination of guanidine compounds in the urine is, with our present available methods, not strictly quantitative. At best one can recover about 90 per cent of added guanidine compounds from watery solution or urine, and this value is only obtained by repeated fractional crystallization, which is a rather tedious procedure not lending itself to work on a clinical scale. For the present purpose, however, the method was sufficiently quantitative.

In view of the recent observations by Major and his co-workers which suggested a relationship between the diminished urinary excretion of guanidine compounds and hypertension, a series of observations were made on normal individuals and on patients suffering from hypertension.

In normal individuals marked fluctuations were found in the daily amounts of guanidine excreted. Two possible explanations of this are suggested. Like most other nitrogenous substances excreted in the urine, the daily amounts may vary. In view, however, of the constancy of creatinine excretion and the chemical relationship between this compound and methyl guanidine, it is quite possible that the total amount of guanidine bases excreted daily is constant for the individual, but that the relative proportions of the different compounds vary and that not all of these are recovered quantitatively by the method employed. 
Our results on the whole are somewhat similar to those of Major. In one case in which daily studies were made, the decrease in blood pressure during the early period of the patient's rest in bed was not accompanied by an increased excretion of guanidine. Later when polyuria was induced and the blood pressure fell still further, excretion of guanidine compounds increased. This suggested a relationship between polyuria and the excretion of guanidine compounds.

An interesting observation was that in the series of cases with hypertension, the majority of those patients who had high diastolic blood pressure also showed diminished excretion of guanidine, whereas those with high systolic but normal diastolic blood pressure had normal excretion of guanidine.

In view of the above findings we are warned that much more must be known of the relationship between guanidine metabolism and hypertension. The results are, however, of sufficient interest to stimulate further work along these lines.

Effects of Roentgen Ray Therapy in Rheumatic Heart Disease. By RoBERT L. LEvy and (by invitation) Ross Golden, New York.

Cardiac involvement in rheumatic fever is frequent and of serious import. No method for its prevention is known, nor has it been possible to modify the progress of lesions initiated in the heart by the rheumatic process.

Radiotherapy is known to influence favorably certain forms of infection; it has also been employed to affect the physical character of scar tissue. These two types of lesion-exudative and proliferative-exist in the heart as the result of rheumatism.

Seven cases of rheumatic heart disease have received courses of roentgen ray therapy, two of them having been followed for one year. The dosage was calculated to yield about 10 per cent of the theoretical erythema dose in the region of the mitral valve.

Clinical improvement has been associated with striking changes in the form of the electrocardiogram in four cases. The remaining cases have thus far received insufficient treatment to warrant a report. The modification of the form of the electrocardiogram apparently associated with roentgen ray therapy suggests that an influence, presumably favorable, has been brought to bear upon the lesions in the heart muscle.

A limited number of roentgen ray treatments has been given to two patients with streptococcus viridans endocarditis without evident effect on clinical course or electrocardiogram.

The Prognostic Value of the Diazo Reaction in Chronic Nephritis. By Regrnald Fitz and (by invitation) HARRY BLOTNER, Boston.

Further Observations on Patients with Hypertension and Increased Basal Metabolic Rate. By ERNST P. BoAs and (by invitation) S. SHAPIRO, New York.

Patients with organic heart disease do not show increased heat production even 
when their cardiac reserve is poor, if the determination is made under actual basal conditions. Only when they exhibit dyspnea at rest do they show increased heat production, and this is readily explained by the muscular exertion and mental distress accompanying the difficult respiration. We examined twenty-eight patients with essential hypertension and two with chronic glomerulonephritis and hypertension under basal conditions. None of them was dyspneic at rest. The patients with glomerulonephritis had normal heat production. Ten patients with essential hypertension exhibited basal rates above plus 15 . Two of nine males and eight of nineteen females showed increased heat production. The distinguishing clinical features of these patients with high basal rates are hypertension, tachycardia, loss in weight, pigmentation of the skin and nervousness. They do not present the picture of Graves' syndrome. Partial thyroidectomy does not seem to improve their clinical condition, and iodine administration does not alter their metabolism. High diastolic pressures are not seen in Graves' disease. We believe that our observations warrant the conclusion that these patients present a special syndrome which should be distinguished from the ordinary form of hypertension as well as from Graves' Disease.

The Significance of Electrocardiograms of Low Voltage. By Howard B. SPRAGUE (by invitation) and PAUL D. WHITE, Boston.

The amplitude of the QRS waves of the human electrocardiogram is probably roughly indicative of myocardial power in cases in which the excursion is less than $5 \mathrm{~mm}$. from the base line. A series of clinical cases is presented in which the electrocardiograms are correlated with the etiology of the heart disease, with the physical findings, and with the prognosis. The most important groups are the cases of arteriosclerosis, and those with myxedema or cretinism. Diminution in the intensity and change in the quality of heart sounds are frequent findings. Aberration of the electrical complexes with changes in the $T$ wave are very common.

Electrical waves of low voltage, except in myxedema and cretinism are usually a bad prognostic sign. They are often found in serious angina and may accompany coronary occlusion. Only 10 per cent of the total cases in our series are able to be actively at work.

Intermittent Incomplete Bundle Branch Block. By F. A. Wrilus (by invitation) and Norman M. Keith, Rochester, Minn.

Three cases are reported, each differing somewhat in type and degree, in which electrocardiograms have been obtained conforming to those of incomplete bundle branch block. In all instances the graphic changes have been present intermittently. These cases are fully discussed with reference to the electrocardiographic abnormalities. The literature on this subject is also considered.

Experimental Study of the Synovial Fluid of Patients with Arthritis and Syphilis. By Alan M. Caesney and (by invitation) Jarold E. KeMP, Baltimore.

Joint fluid was aspirated from a series of ten patients in various stages of 
syphilis, in whom arthritis was present in addition. The fluids were studied with reference to $(a)$ cytology, $(b)$ Wassermann reaction, $(c)$ presence of bacteria by smear, culture and guinea pig inoculation, $(d)$ presence of Treponema pallidum by dark field examination and intratesticular inoculation in rabbits.

Virulent strains of $T$. pallidum were obtained by rabbit inoculation from three of the ten joint fluids studied. A relatively high lymphocytosis was encountered in two of these fluids as well as in two others in which there was reasonably good evidence that the arthritis was syphilitic in origin. Fluids from those cases which were believed to be non-syphilitic did not show this relative increase in lymphocytes.

The Physiology of the Leucocytes in the Saliva in Health and Disease. By RaPHAEL IsAacs, Boston.

Quantitative studies of the leucocytes of the blood and of the saliva show that when the leucocytic blood count rises, the number of leucocytes in the saliva tends to decrease; and when the leucocytic blood count decreases, the saliva count increases. Normally, the saliva collected from the salivary ducts has few or no leucocytes. When the saliva is allowed to collect in a pool in the mouth it washes together leucocytes which have wandered through the mucous membrane. The number varies from five or less per cubic millimeter to about 150 , but may reach 400 in health. When a "digestive leucocytosis" fails to show in the blood, it is frequently replaced by an increase in the number of leucocytes in the saliva. All types of leucocytes appear in the saliva, and they live for varying lengths of time dependent on the disease or the therapy. In chronic myelogenous leukemia, the saliva count may be surprisingly low when the blood count is high. After roentgen ray treatment the saliva cell count increases, at times to 10,000 or more per cubic millimeter, with the decrease in the blood leucocyte count. In aleukemic leukemia the saliva cell count may be very high.

The Treatment of Paroxysmal Auricular Tachycardia. By S. A. Levine and (by invitation) HARRY BLOTNER, Boston.

The Onset of Digitalis Effect after Intravenous Administration. By HAROLD E. B. PARdeE, New York.

The effects of digitalis which were observed in these experiments were: (1) the slowing of ventricular rate in patients with rapid rates from auricular fibrillation, (2) the diminution in size of the $\mathrm{T}$ wave of patients with normal rhythm.

Digitan solution and Digalen were the chief preparations used. These were given intravenously in a dose of 1 minim of the solution per pound of body weight of the patient. In the patients with auricular fibrillation the heart rate was counted with a stethoscope several times for one-half minute periods to obtain a control rate and then at various intervals during the next 24 hours. In those with normal rhythm a control electrocardiogram was taken before the drug was given, and then at proper intervals for 24 hours. 
It was found that the effect upon rate often began as soon as $\mathbf{5}$ minutes after intravenous administration of these doses, always within 15 minutes. The slowing progressed rapidly during the first 30 minutes and somewhat less rapidly during the next hour. The maximum slowing was usually 2 hours after administration but most of the effect developed during the first hour. After reaching its maximum the effect persisted, diminishing slightly, but even 24 hours after the dose, the rate had not reached its control level.

The effect upon the $T$ wave also appeared as soon as 5 minutes after the dose and reached its maximum about 2 or 3 hours later, then passed off gradually.

The important results of these experiments are to show that the doses of these preparations previously suggested by the author are proper for clinical use; that the onset of digitalis effects after proper intravenous doses is more prompt than was previously believed and that the effect upon rate and the effect upon the muscle proceed at about an equal rate.

Rickets in Dogs. By Alfred T. ShoHL, New Haven.

Observations on the pathogenicity of Brucella abortus for human beings. By ERNEST

C. Dickson, San Francisco.

Following an illness in January, 1925, Miss C. was advised to drink plenty of milk. From February 15 to June 7 she consumed from four to six glasses of milk daily, part of it being pasteurized and the remainder being raw milk from an inspected dairy.

In the latter part of May she developed an intermittent fever associated with sweating, fatigue and pains in the legs. The fever persisted for several weeks, ranging from 99 to 101 or 102 degrees daily with occasional remissions. The only notable physical sign was a palpable spleen.

On July 3, the patient's blood serum caused complete agglutination of $\mathrm{Br}$. melitensis in dilution of 1:500 and partial agglutination in 1:1000. Subsequently Miss Alice Evans at Washington reported that it was the Br. abortus variety.

Investigation of the dairy showed that there had been little trouble with infectious abortion although two cows had aborted within fifteen months. Whey prepared from the milk of these cows caused agglutination of $\mathrm{Br}$. abortus in dilutions of $1: 100$ and $1: 200$, respectively, and guinea pigs which were injected with the milk developed typical lesions of $\mathrm{Br}$. abortus infection from which $\mathrm{Br}$. abortus was recovered in pure culture. 